\title{
Virtual Reality
}

\section{Digital preservation and display of the national cultural heritage}

\author{
LI Jianyang \\ College of Computer Science and Technology, SWUN \\ Chengdu, Sichuan \\ e-mail: $\underline{81185591 @ q q . c o m}$
}

\begin{abstract}
The digitization of the national cultural heritage and relics are actualized through virtual reality technology. Research on the protection and display of digitization of the national cultural heritage lies in the 3D scanning techniques, 3D color model and 3D texture mapping, which explains the operational principle. Scanning the relics through the 3D scanner, the denoising and splicing of data and the post - processing of texture mapping result in 3D digital models. 3D scanning technology is used to research the basic process and method of digital cultural relics.
\end{abstract}

Keywords-virtual reality; digitization; 3Dscanning techniques; $3 D$ color model; texture mapping

China's thousand-year-long history provides the Chinese nation and the world with a precious cultural heritage including the tangible cultural heritage of scenic spots and historical sites and rare relics and the in tangible cultural heritage of dancing, poetry and mythology. Intangible cultural heritage can be preserved through a variety of ways, such as books, audio, video etc. However, with the fleeting of time and the impact of human activity, a lot of tangible cultural heritages have been destroyed. How to use advanced technology to protect these cultural heritage has been imminent. the rapid development of computer technology and the rising of virtual reality technology bring a new dawn for cultural heritage -the highprecision and high-fidelity digital heritage protection technology of cultural heritage ${ }^{[1]}$.

\section{THE PROTECTION AND DISPLAY OF THE VIRTUAL REALITY AND DIGITALIZATION OF CULTURAL RELICS}

Virtual reality (Virtual reality, VR), as a 3D-virtual-spaced analog system through which to be able to create and experience virtual world, is a $3 \mathrm{D}$ environment created by computer to produce such a feeling of indulging in virtual environment through human's function of vision, audition and tactile sensation that humanizes human-computer communication and enables users to interact directly with information.

The protection and display of digitization of the national cultural heritage firstly, lies in digital photography, 3D scanning and 3D color model through which heritage entities can be used to set up a substantial 3D or model database to preserve the important resource of relation between original

Supported by the central fund for young college teachers. (13NZYQN15) LI Jianyang (1979- ), Research Assistant,

Research direction: computer simulation, database

\author{
RUAN Sishi \\ College of Computer Science and Technology, SWUN \\ Chengdu, Sichuan \\ e-mail:276807646@qq.com
}

various data and space of relics and actualize scientific, highprecision and the permanent preservation of the realization of rare heritage resources. Secondly, making use of virtual reality technology through which network makes overall exhibition of heritage more realistic. It can become cultural heritage possessed by all human. The digitalization of heritage not only enables the 3D expression and virtual display. More importantly, accurate digital model of heritage record the true 3D and texture information of original heritage, providing important data and model support for the repair and restoration of heritage $\mathrm{e}^{[2 \sim 3]}$.

This paper will discuss on the technology relating to Digital cultural relics protection and display of digitization of the heritage, among them 3D scanning techniques is one of the means widely used in the digitization of the heritage ${ }^{[4]}$.

\section{3D SCANNING TECHNOLOGY}

\section{A. The working principle of $3 D$ scanner}

The core principle of 3D laser scanning is laser ranging. Laser light irradiate to the surface of the measured object through 3D laser scanner send a line and collect the reflection information. Measuring the distance of the multi-point in the surface of scanned object, and obtain the 3D data of the cloud point relative position from the object surface.

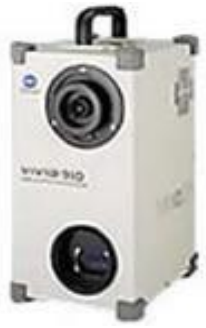

Figure 1. Vivid 910 3D scanner

Then processed by computer software, rebuilding the model of point, line and solid rapidly. Laser scanning equipment is divided into two: contact and non-contact. We commonly use non-contact 3D laser scanner for heritage scanning. The data obtained by scanner are in the form of point, because of the number of points is enormous and organized for each scan. This set of points is vividly called the "Point Cloud". Point cloud file can be sliced browsing, surface treatment and 3D modeling to meet the needs of data processing person ${ }^{[5]}$. 


\section{B. Based on 3D laser scanning technology to obtain 3D model of heritage}

Based on the current 3D scanning technology we could get historical relic's apparent point cloud data so that we able to work out its CAD stereoscopic model through some post processing. Or mapping on the 3D saner by the technology of image processing in order to make the digital models of historical relic more vivid. Not only could reserve them permanently or work for research, but also could put into use on the historical relic's virtual exhibition.Mainly including these following tips:

1) Data collecting Original points point cloud data gain from the 3D laser scanner generally. It's worthy nothing that environment and the size of historical relic often limit to the process. First of all, putting the equipment on the control point in order to accomplish the directional block surface. Besides, you could adjust the historical relic if it small enough. There have no possible that we gain the complete in formations at a time, so we must check it again and again. In order to joint them expediently, we always collect data by turn the object 45 degree Angle when we attempt to collect data till we could gain all the surface information of the historical relic.

2)Data Processing The raw point data obtained through the 3D laser scanner is very large, also there exit noise data. According to modeling requirements, we need to remove the noise in the data point, so that we can obtain follow up data required for modeling process.

The template is used to format your paper and style the text. All margins, column widths, line spaces, and text fonts are prescribed; please do not alter them. You may note peculiarities. For example, the head margin in this template measures proportionately more than is customary. This measurement and others are deliberate, using specifications that anticipate your paper as one part of the entire proceedings, and not as an independent document. Please do not revise any of the current designations.

a)Data de-noising Due to the external environment and instrument itself and many other factors, requiring data often produces noise points and easily makes errors in the modeling process. So there is no doubt that we must eliminate these noise points in the data processing. As for the obvious noise points, generally, we can directly remove them through the judgment of naked eyes, while those inconspicuous noise and the noise of instrument itself can be identified by the intensity of laser scanning echo signal. if the intensity of echo signal is lower than the threshold, the distance signal value will be invalid. Utilizing the median filter, we can take singular points out of it. After that, we can make use of the surface fitting to kick the front obstruction. According to different conditions, Cloud Point de-noising works out corresponding handle, especially the way the combination of manual operation and software to get rid of the noise points.

b)Data splicing

Adopting three point fixing, when we match the two cloud points, picking out three featured data points from the two-data coincide fragment respectively. While selecting, we should avoid the three points on the same line or coordinate plane to ensure the data-joint precise. Feature point can selected by the surface characteristics, if necessary, making the feature signs before scanning, which is helpful for accurately ensure the centralized common points between the two cloud point and enhances the data precise in the tiling fitting.

\section{THE ACHIEVEMENT OF CULTURAL RELICS 3D COLOR MODEL}

For the digital information of cultural relics, only relying on 3D digital model can not be enough to achieve cultural relics 3D color model; we need to record its color. Namely, we should not only get the geometrical information of cultural relics, but also get the color texture information of its surface, so 3D digital modal can correspond to the color. The texture, put simply, is the color information of the grid model's surface. When grid model is formed by meshes, texture is the collection of colors corresponding to every mesh's vertex. In order to obtain high resolution and precise 3D model of cultural relics, the emphasis should be put on the texture mapping between grid model and image, namely, establishing the correspondence between every vertex of grid model and image pixel ${ }^{[6]}$. Integrating the traditional way of recording the cultural relics information and present way of reproducing 3D model and color texture, there are two ways as follow: the first is using specialized hardware equipment to get aggregation model and color picture of the object under the same viewpoint, thus obtaining the natural texture mapping; the second is to collect 3D model and texture respectively, then fitting them later.

\section{A. Equipment with texture processing mode}

Now, there are many 3D scanners, which can record the color and process the model and texture at the same time. Like American FARO Laser Scanner, Japanese Konica Minolta 3D Laser Scanner, Swiss Lei-ca 3D Laser Scanner, French Men-si and so on, they all have that function. When gathering 3D image of cultural relics, using a camera lens can get geometric and color information at the same time, also it can map the material to the corresponding geometry. Thus, finally resulting 3D grid model, actually, has texture information, namely, every vertex of grid model owns a color value. However, the drawback of it is that restricted to the condition of hardware equipment, texture pixels are not high and can not meet the visual demand of high definition. Meanwhile, strict experimental environment should be set up, otherwise, the light conditions are difficult to be the same when taking color images at different viewpoint, and the luminance of these color images will not be all the same. When different images, finally, are mapped to the surface of complete grid model, probably in the joint area of images, there are obvious inconsistent textures on visual effects appearing, thus impacting the effect. And magnifying the 3D model will cause distortion and can not meet the demand of high precision.

\section{B. The fitting of color texture in the later stage software}

Specialized graphics processing software (like 3D Max) should be used to do the texture mapping. After data collection and processing, we get the cultural relics 3D model, making use of the function of multiple texture mapping in 3D Max, 
with manual interactive mode, putting many graphics onto one model, doing the texture mapping for the model ${ }^{[7]}$.

\section{1) Manual mode}

Manual fitting way is to shoot precisely the color texture of the processed object with the way of high-definition digital photography, then doing the precise manual fitting by 3D specialized software, unifying texture and model. The advantage of it is that color information of high definition can be obtained; the difficulty is that it's hard to choose the photographic methods of high definition texture images and accurate positioning of texture depends on the experience and technique of operating staff during the texture and model fitting process in the later stage software.

\section{2) Semi-automatic and automatic mode}

Semi-automatic and automatic mode is to do the fitting of obtained color image texture and 3D model by the computer process mode. Like the clay model of Zhejiang University and Dunhuang Academy, they adopt that method for the model. They have researched the way to get the model through 3D scanner and an object's texture mapping through a series of digital photographs. The algorithm obtained by the model image alignment technique, projection and geometric distortion and stitching and other steps to obtain the cylindrical object textures, which has the advantage of Intuitive, the original photo quality requirements, and allowing the runtime editor and so on.

\section{Perspective projection model}

Because of these methods have flaws and shortcomings, we hope to design an efficient algorithm which can build the texture mapping between grid model and the color image automatically or semi-automatically based on some clues of grid model and color images. Cultural heritage grid model is the representation in the world coordinate system of reality heritage that discrete and sampled through 3D scanner. According to the perspective projection model, firstly, change it's coordinate to the captured color image of the camera where the camera coordinate system. Then, change it's perspective projection to the image coordinate system to the plane. Thereby generating a two-dimensional color image of heritage. This is actually a match problem between the images and the grid model. We label the corresponding feature of some images and the grid model by hand. Use the perspective projection model to match the images and grid model. Thereby solving the texture mapping of images and the model ${ }^{[8]}$.

Texture mapping process is as follows:

\section{1) Calculation of the perspective projection matrix}

Let one point $\mathrm{P}$ of the heritage grid model has the world coordinates $(\mathrm{x}, \mathrm{y}, \mathrm{z})$, the corresponding point coordinate of point $\mathrm{P}$ in a color image is $(\mathrm{u}, \mathrm{v}),(\mathrm{x}, \mathrm{y}, \mathrm{z})$ and $(\mathrm{u}, \mathrm{v})$ is called a set of feature correspondences. The relationship between them can expressed in homogeneous coordinates and matrix form:

$$
\left[\begin{array}{l}
u \\
v \\
1
\end{array}\right]=M\left[\begin{array}{l}
x \\
y \\
z \\
1
\end{array}\right]
$$

$M=\left[\begin{array}{llll}m_{11} & m_{12} & m_{13} & m_{14} \\ m_{21} & m_{22} & m_{23} & m_{24} \\ m_{31} & m_{32} & m_{33} & m_{34}\end{array}\right]$ is the Perspective projection matrix of Equation (1). Solving the perspective projection matrix $M$ by manually marking the corresponding feature points of multiple grid model and corresponding image.

\section{2) Seeking texture values of every grid point in mesh}

After perspective projection matrix $M$ is obtained, traverse all points on the grid model. For each point, obtain the corresponding coordinate point in the two-dimensional image by equation(1). And assign the RGB value of the twodimensional image to the point on the grid model as the texture values of the grid point.

\section{Case Study of Heritage Digital Solutions}

We take digitization process of heritage which shown in Figure 2 for an example.
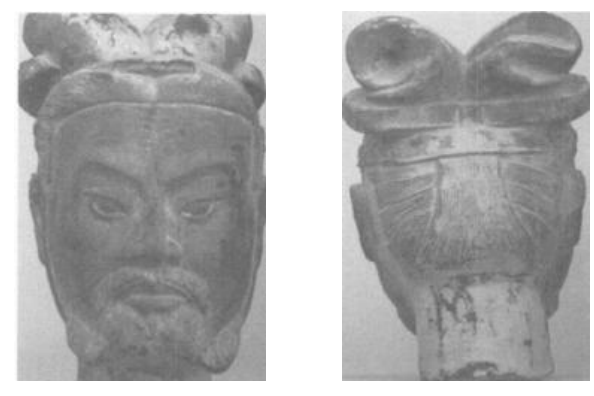

Figure 2. Real photos of heritage

First, collect the space data and texture data of heritage. Then, integrate all the 3D data, generate 3D grid model.Finally, establish the precise digital model through texture mapping.

\section{A. 3D modeling of heritage}

First, collect the space data and texture data of heritage by Vivid 910 3D laser scanner and high-resolution digital camera. The scan of heritage can be repeated. To ensure the accuracy of splicing, adjacent scan should be made with a certain degree of overlap. Then, pretreat the 3D data, data de-noising, data splicing, Generate 3D grid model. 


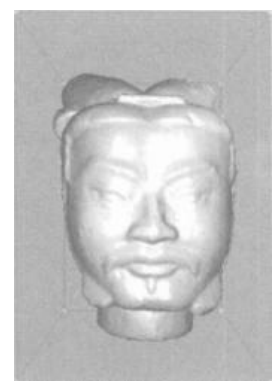

Figure 3. Scan data of heritage

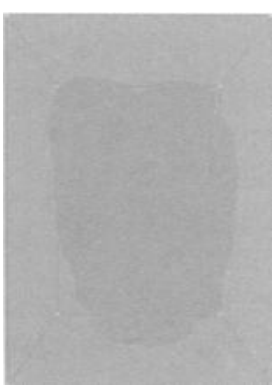

Figure 4. Grid model

\section{B. Textures}

In order to obtain high accuracy Texture while scanning model, we fixed the high-resolution digital camera above the Vivid 910. Obtain the corresponding high-resolution image while scanning. Pay attention to eliminate the impact of high light when shooting. Here, we adopt the artificial texture mapping scheme. The 3D models after texture are shown in Figure 5.

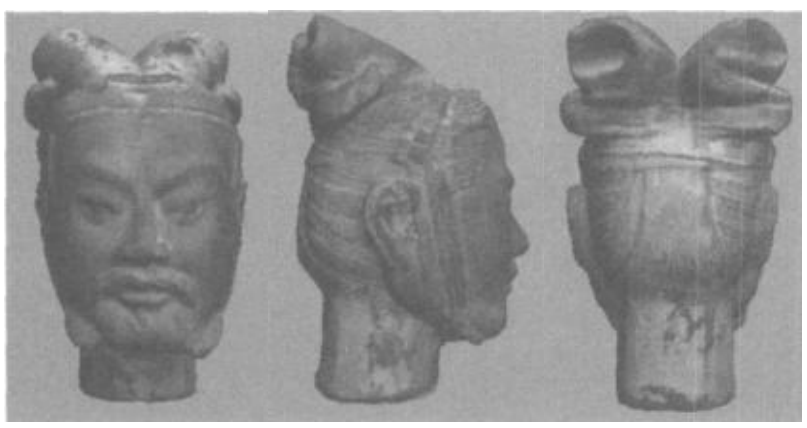

Figure 5. Heritage digital model after mapping

\section{Virtual exhibition of heritage}

The established 3D models of heritage can be published on the web for virtual exhibition. Users can freely control the 3D models through the mouse and keyboard.Also the 3D models can be processed into physical artifacts by rapid molding machine. Achieve true representation of heritage.

\section{CONCLUSION}

The role of virtual reality technology on national cultural heritage such as culture relics is self-evident and virtual reality technology has played an invaluable role on heritage conservation, display and recovery process. Using 3D scanning technology and 3D color model implements digitization of cultural relic, get high-quality digital model. After several years of development, the 3D digital technology of cultural relic has made a lot of research. But there are many issues that need research to improve.

(1)We need further study on the point cloud data processing(such as data smooting, data de-noising).

(2)Study efficient Semi-automatic and fully automatic texture mapping algorithm, improve the accuracy and speed of Texture mapping 3D models, achieve bulk 3D digital of heritage.

\section{REFERENCES}

[1] HU Weixi, PAN Zhigen, LIU Xizuo, etc. Key Technical Overview of Virtual World Natural and Cultural Heritage Protection [J]. Journal of System Simulation. 2003, 15(3): 315.

[2] ZHANG Aiwu, HU Shaoxing, SUN Weidong, etc. Outdoor Scenes 3D Reconstruction Based on Laser and Visible Light Synchronize Data [J]. Electronic Journal. 2005, 33(5): 810-815.

[3] HU Shaoxing, CHA Hongbin, ZHANG Aiwu. 3D Digital Method of Large-scale Ancient Heritage [J]. Journal of System Simulation. 2006 , 18(4): 951-954.

[4] TIAN Ye. The Road of Museum Digitization [N]. Guangming Daily. 2007-01-17G.

[5] ZANG Chunyu. The Application of 3D Laser Scanning Technology in Cultural Relic Protection Research [J]. Architectural Journal. 2006.

[6] ZHANG Yingjie, ZHANG Tiechang. Survey Staves of Texture Mapping . Computer Engineering and Applications. 1994, 3(4): 1-4.

[7] HUANG Xinyuan. Standard Tutorial of 3ds Max 6 [M]. Beijing: People Post Press. 2004.

[8] ZHAO Bing. Conservation-oriented Texture Mapping Method [D]. Peking University College of Information Science. 2005: 5.

[9] QIU Zhaowen, ZHANG Tianwen. Key Technology of Heritage 3D Reconstruction [J]. Electronic Journal. 2008, 36(12): 2423-2427.

[10] JIA Hongyu, LV Zhipeng. Digital Research and Practice of Heritage Based on 3D Scanning [J]. Journal of Yangtze University. 2009, 6(3): 253-255. 